\title{
Deficit of Learning and Memory in Children with Hiperactivity and Attention deficit (ADHD). A Clinical Descriptive and Qualitative Study
}

\author{
Castejón 0J*, Torres IA, Galindez P, Villasmil A, Grumbaum E and Salones de Castejón M \\ Faculty of Medicine, Venezuela
}

*Corresponding author: Orlando J Castejón, Faculty of Medicine, Venezuela

\begin{abstract}
ARTICLE INFO
Received: May 20, 2019

Published: May 21, 2019

Citation: Castejón OJ, Torres IA, Galindez P, Villasmil A, Grumbaum E, Salones de Castejón M. Deficit of Learning and Memory in Children with Hiperactivity and Attention deficit (ADHD). A Clinical Descriptive and Qualitative Studyb. Biomed J Sci \& Tech Res 18(2)-2019. BJSTR. MS.ID.003115.
\end{abstract}

Keywords: Cognitive Deficit; Learning Deficit; Memory Deficit; ADHD

\author{
ABSTRACT
}

We have clinically examined 40 patients with ADHD ranging from 3 to 12 years-old, exhibiting attention, learning and memory deficit. During mother pregnancy we found urinary infections, edema of lower limbs, arterial hypertension, diabetes, eclampsia and placental pathology. The child social relationships and academic performance at the school and home was explored by means of dialogs with the mothers or relatives, teacher information and psych pedagogical study. A previous psychological study of patients was carried out at private and public psychological centers of Maracaibo City in Venezuela. The infant patients showed hyperactivity, impulsivity, inattention, learning and memory deficit, neurosensory sensory processing difficulties, such as hypoacusis and strabismus, inappropriate behavioral impairment, such as emotional instability, anxiety, sleep disorders and auto and heteroaggression. Hyperactivity included an excessive rate of speech and motor activity or motor restlessness and being easily distracted.

Abbreviations: ADHD: Attention-Deficit Hyperactivity Disorder; SWS: Slow Wave Sleep; SDB: Sleep-Disordered Breathing; SDB: Sleep Disordered Breathing; PFC: Prefrontal Cortex; LC: Locus Coeruleus; EF: Executive Function; NMDARs: N-methyl-d-aspartate receptors NMDARs; BPD: Borderline Personality Disorder; NET: Norepinephrine Transporter; LD: Learning Disability; WM: Working Memory

\section{Introduction}

Children suffering from attention-deficit hyperactivity disorder (ADHD) often also display impaired learning and memory. Previous research has documented aberrant reward processing in ADHD as well as impaired sleep-dependent consolidation of declarative memory. Studies of ADHD children point toward an essential contribution of prefrontal cortex to the preferential consolidation of declarative memory during slow wave sleep(SWS) [1], According to Prehn Kristensen et al. [2] functional magnetic resonance images show prefrontal hypoactivity. They have formulated the hypothesis that children with ADHD benefit from sleep with respect to procedural memory more than healthy children. According to these Authors, children with ADHD showed an improvement in motor skills after sleep compared to the wake condition.

These data suggest that sleep in ADHD normalizes deficits in procedural memory observed during daytime, ADHD children could present sleep problems such as agitated sleep, sleeponset difficulties, low arousal threshold during the night. Such sleep difficulties have been shown to occur more frequently in children with severe diurnal ADHD symptoms. Prevalence and physiopathology of sleep disorders including awakening mechanisms (micro-arousals, arousal threshold) from sleep yet need to be clarified in ADHD children. Excessive nocturnal motricity could be the expression of a monoaminergic dysfunction previously reported in ADHD and could lead to new therapeutic gateways as well as hypo vigilance Lecendreux et al. $[3,4]$ examined the hypothesis that domains of neurobehavioral function would be selectively affected by sleep-disordered breathing (SDB).

The Authors assessed potential relationships between objectively measured sleep disturbances and neurobehavioral function in children with reported symptoms of attention-deficit/ 
hyperactivity disorder (ADHD) and also determined the incidence of snoring and other sleep problems in 5- to 7-year-old children in the local community and potential relationships to parental snoring and passive smoking. According to Gottlieb et al. [5] sleepdisordered breathing (SDB), hyperactivity and inattention are common with aggressiveness and daytime sleepiness symptoms in 5-year-old children and are associated with an increased risk of daytime sleepiness and with problem behaviors suggestive of ADHD. Maternal prenatal anxiety is an important risk factor for altered child neurodevelopment but there is uncertainty concerning the biological mechanisms involved and sources of individual differences in children's responses Kieran et al. [6,7]. In the present study we examined from the clinical point of view the learning and memory deficits in infant patients, 3 to 12 years-old, with hyperactivity and attention deficit (ADHD).

\section{Material and Methods}

We have clinically examined 40 patients with ADHD, ranging from 3 to 12 years old, attending the Neuroscience Outpatient Clinic in the San Rafael Home Clinic of Maracaibo. Venezuela, exhibiting inattention, learning and memory deficits. The mother pregnancy was explored looking for urinary infections, edema of lower limbs, arterial hypertension, diabetes, eclampsia, placental pathology and conditions of fetal delivery. The affecting relationships and academic performance at the school and home was explored by means of dialogs with the mothers or relatives and psych pedagogic studies carried out at the school. A previous psychological study of patients was carried out at private and public psychological center of Maracaibo City in Venezuela.

\section{Results}

The infant patients showed hyperactivity exhibiting mainly attention, learning and memory deficits. Some patients exhibited sensory processing difficulties, inappropriate behavioral impairment, such as emotional instability, anxiety, insomnia, impulsivity and auto aggression and heteroaggression. Hyperactivity included an excessive rate of speech and motor activity or motor restlessness and being easily distracted. The findings strongly suggest impaired sleep-dependent consolidation of declarative memory.

\section{Discussion}

Attention-deficit/hyperactivity disorder (ADHD) is a neurobiological condition of childhood onset with the hallmarks of inattention, impulsivity, and hyperactivity. Inattention includes excessive daydreaming, disorganization and being easily distracted. Impulsivity manifests as taking an action before fully thinking of the consequences. Hyperactivity includes an excessive rate of speech and motor activity. Complications of ADHD include academic failure, low self-esteem, poor work performance, substance abuse, criminal justice issues and social problems Bokor et al. [8]. Learning and memory deficits observed in our patients are related with perinatal hypoxia and above described mother pathological conditions during pregnancy. A cascade of cellular events including reduction of intracellular calcium levels, reduction of brain derived neurotrophicfactor release, neuronal cell death, decreased glutamate presynaptic release and aberrant dendritic morphology occur at the brain parenchyma. The above-mentioned events lead to decreased synaptogenesis and decreased neuronal network formation and function causing learning and memory deficit at organism level 0 Donnell et al. [9] Some neurodevelopmental alterations, such as aberrant nerve and glial cell migration, formation of abnormal nerve cell circuits, synaptic plasticity and degeneration can take place in in pre- and postnatal conditions Castejón et al. [8,10].

We observed patients with sleep disorders which suggest an alteration of memory consolidation process during sleep. Diekelmann et al. [11] hypothesize that access to sleep-dependent consolidation requires memories to be encoded under control of prefrontal-hippocampal circuitry, with the same circuitry controlling subsequent consolidation during sleep. Sleep supports the consolidation of declarative and procedural memory. While prefrontal cortex (PFC) activity supports the consolidation of declarative memory during sleep, opposite effects of PFC activity are reported with respect to the consolidation of procedural memory during sleep Wilhelm et al. [12]. Patients with attentiondeficit/hyperactivity disorder (ADHD) are characterized by a prefrontal hypoactivity, which explain inattentive behavior and deficit of memory. Many ADHD patients have cognitive dysfunction and sleep problems, including longer sleep latency, lower sleep efficiency, and shorter total sleep time Lee et al. [13]. The vast majority of attention-deficit/hyperactivity disorder (ADHD) patients have other associated pathologies, with depressive symptoms as one of the most prevalent. Among the mediators that may participate in ADHD, melatonin is thought to regulate circadian rhythms, neurological function and stress response Cubero-Millán et al. [14]. In addition to, system-level, local and cross-regional interactions, a consolidation mechanism involves stabilization of memory representations at the synaptic level. Synaptic plasticity within experience-activated neuronal networks is facilitated by noradrenaline release from the axon terminals of the Locus Coeruleus (LC) Novitskaya et al. [15,16].

consider that deficits in executive function (EF) underlie the core neuropsychological sequelae of attention-deficit/hyperactivity disorder (ADHD), particularly deficits in working memory and inhibitory control arising from dysfunction in the prefrontal cortex. Some infant patients exhibited neurobehavioral disorders such as emotional instability. According to Banich et al. [17] the neural mechanisms involved in cognitive control over memory processes that can influence, and in turn are influenced by emotional processes. With regard to working memory, these Authors discuss the neural bases of control mechanisms that can select against 
distracting emotional information, mechanisms that can regulate emotional reactions or responses, how mood state influences cognitive control and individual differences in control mechanisms. For long-term memory, they briefly review the neural substrates of emotional memory, the cognitive and neural mechanisms that are involved in controlling emotional memories and how these systems are altered. Recent pediatric studies have suggested a correlation between decreased amygdala volume and attention deficit and hyperactivity disorder (ADHD) symptoms, including the emotional dysregulation.

The findings are consistent with the possibility that hyperglutamatergic processes in the amygdale are related to the hyperactive-impulsive symptoms of ADHD Wiguna et al. [18]. We have above reported impulsivity in most patients examined. Disorders such as borderline personality disorder (BPD) or attention-deficit/hyperactivity disorder (ADHD) are characterized by impulsive behaviors. Impulsivity as used in clinical terms is very broadly defined and entails different categories including personality traits as well as different cognitive functions such as emotion regulation or interference resolution and impulse control Sebastian et al. [19]. According to Sachana et al. [20] chronic binding of antagonist to N-methyl-d-aspartate receptors (NMDARs) during brain development induces impairment of learning and memory abilities of children. In our study, we have also found sensory processing impairments. According to Shimizu et al. [21]. children with ADHD may present sensory processing impairments, which may contribute to the inappropriate behavioral and learning responses displayed by children with ADHD. It also suggests the importance of understanding the sensory processing difficulties and its possible contribution to the ADHD symptomatology. Visual attention functions as a filter to select environmental information for learning and memory, making it the first step in the eventual cascade of thought and action systems. interactions between visual processing and visual attention, as well as the contribution of visual attention to memory Amso et al. [22].

Deficits in executive function (EF), impaired school functioning and altered white matter integrity in fronto-striatal networks have been associated with attention-deficit/hyperactivity disorder (ADHD). An association between white matter integrity in the fronto-striatal networks and school functioning and suggest that $\mathrm{EF}$ deficits and ADHD symptoms may be the mediating mechanisms for this association. Future research is needed to test the directionality and specificity of this finding Gau et al. [23]. Attention-deficit/ hyperactivity disorder (ADHD) research has long focused on the dopaminergic system's contribution to pathogenesis, although the results have been inconclusive. However, a case has been made for the involvement of the noradrenergic system, which modulates cognitive processes, such as arousal, working memory, and response inhibition, all of which are typically affected in ADHD. Furthermore, the norepinephrine transporter (NET) is an important target for frequently prescribed medication in ADHD.
Therefore, the NET is suggested to play a critical role in ADHD Ota et al. [24]. Recent studies have shown that ADHD is characterized by multiple functional and structural neural network abnormalities including most prominently fronto-striatal, but also fronto-parieto-temporal, fronto-cerebellar and even fronto-limbic networks Castejon et al. [25] Evidence from longitudinal structural imaging studies has shown that ADHD is characterized by a delay in structural brain maturation Rubia et al. [26]. The anomalies were found in tracts that connect components of neural systems pertinent to ADHD, such as attention control (inferior frontooccipital fasciculus) and emotion regulation and the processing of reward (the uncinate fasciculus). Change in radial rather than axial diffusivity was the primary driver of this effect, suggesting pathophysiological processes including altered myelination as future targets for pharmacological and behavioral interventions Shaw et al. [27].

According to Hong etal. [28] ADHD patients showed significantly reduced dorsal caudate functional connectivity with the superior and middle prefrontal cortices as well as reduced dorsal putamen connectivity with the parahippocampal cortex. Wu et al. [29] explored the memory characteristic in boys with attention-deficit/ hyperactivity disorder (ADHD) plus learning disability (LD), and postulated that boys with ADHD comorbid LD show deficits in overall memory function and long-term memory while short-term memory is partially damaged. Impairment in immediate memory is not detected. It is important to consider that sleep problems in children with ADHD are commonly transient, but in a subgroup, they are characterized as persistent. Therefore, early preventive/ intervention strategies should target children at risk of persistent sleep problems Lycett et al. [30].

Brain areas generating emotions, such as the amygdala, and those allowing the association of multi-dimensional stimuli into an episodic memory, such as the hippocampus, are delineated. Patients with emotional and affective disorders show changes in memory performance in dependence of the positive or negative valence of the stimulus material. Furthermore, these patients often exhibit a reduced ability to access specific memories of life events with a striking lack of specific detail Dere et al. [31]. The extent to which deficits in working memory (WM) are characteristic of children with reading and mathematics difficulties was investigated in a large sample aged 5-15 years reported to have problems in attention, learning and memory. WM or the broader cognitive dimensions it taps impede school-based learning, and point to the importance of managing WM loads in the classroom Gathercole et al. [32].

\section{Conclusion}

The infant patients showed hyperactivity, impulsivity, aggression, inattention, learning and memory deficit, neurosensory sensory processing difficulties, such as hypoacusia and strabismus, inappropriate behavioral impairment, such as emotional instability, anxiety, insomnia, and aggression. This finding are related with 
maternal pathology and environmental conditions. The neural correlates of clinical findings are described. A correlation between deficit of learning and memory and child sleep disturbance is emphasized.

\section{Acknowledgement}

This investigation has been supported by a subvention from Castejón Foundation. The logistic, personal and administrative resources of San Rafael Clinical Home of Maracaibo and Biological Research Institute, Faculty of Medicine. Zulia University are deeply appreciated.

\section{References}

1. Wiesner CD, Molzow I, Prehn-Kristensen A, Baving L (2017) Sleepdependent consolidation of rewarded behavior is diminished in children with disorder and a comorbid disorder of social behavior. Front Psychol 8: 167-169.

2. Prehn Kristensen A, Munz M, Wilhelm I, Muller K, Freytag D, et al (2011) Transcranial oscillatory direct current stimulation during sleep improves declarative memory consolidation in children with attentiondeficit/hyperactivity disorder to a level comparable to healthy controls. Res. Dev. Disabil 32(6): 2480-2488.

3. Lecendreux M, Konofal E (2002) Sleep and vigilance in hyperactive children. Rev Prat 52(18): 2002-2004.

4. O Brien LM, Holbrook CR, Mervis CB, Klaus CJ, Bruner JL, et al. (2003) Sleep and neurobehavioral characteristics of 5- to 7-year-old children with parentally reported symptoms of attention-deficit/hyperactivity disorder. Pediatrics. 111(3): 554-563.

5. Gottlieb DJ, Vezina RM, Chase C, Lesko SM, Heeren TC, et al. (2003). Symptoms of sleep-disordered breathing in 5-year-old children are associated with sleepiness and problem behaviors. Pediatrics 112(4): 870-877.

6. Kieran JO Donnell, Vivette Glover, Jari Lahti, Marius Lahti, Rachel D Edgar, et al. (2017) Maternal prenatal anxiety and child COMT genotype predict working memory and symptoms of ADHD.

7. Castejón OJ, Galindez P, Torres IA, Leal J, Villasmil A, et al. (2018) Maternal Stress, Pregnancy Diseases and Child Hyperactivity and Attention Deficit. Biomedical Journal of Scientific \& Technical Research (BJSTR) (ADHD)

8. Bokor G, Anderson PD (2014) Attention-deficit/hyperactivity disorder. Pharm Pract 27(4): 336-349.

9. KO Donnell KJ, Glover V, Lahti J, Lahti M, Edgar RD, et al. (2017) Maternal prenatal anxiety and child COMT genotype predict working memory and symptoms of ADHD. Plos One 12(6): e0177506.

10. Castejón OJ, Galindez P, Torres IA, Merchan F, Villasmil A, et al. (2019) Subgroups of Attention Deficit Disorder (ADHD). A preliminary clinical descriptive study Research (BJSTR).

11. Diekelmann S, Wilhelm I, Born J (2009) The whats and whens of sleepdependent memory consolidation. Sleep Med Rev 13(5): 309-321.

12. Wilhelm I, Prehn Kristensen A, Born J (2012) Sleep-dependent memory consolidation-what can be learnt from children? Neurosci. Biobehav Rev 36(7): 1718-1728.

13. Lee HK, Jeong JH, Kim NY, Park MH, Kim TW, et al. (2014) Sleep and cognitive problems in patients with attention-deficit hyperactivity disorder. Neuropsychiatr Dis Treat 10: 1799-1805.

14. Cubero Millan I, Molina Carballo A, Machado Casas I, Fernández López L, Martínez Serrano S, et al. (2014) Methylphenidate ameliorates depressive comorbidity in ADHD children without any modification on differences in serum melatonin concentration between ADHD subtypes. Int J Mol Sci 15(9): 17115-17129.
15. Novitskaya Y, Sara SJ, Logothetis NK, Eschenko O (2016) Ripple-triggered stimulation of the locus coeruleus during post-learning sleep disrupts ripple/spindle coupling and impairs memory consolidation. Noviskaya Learn Mem 23(5): 238-248.

16. Duff CT, Sulla EM (2015) Measuring executive function in the differential diagnosis of attention-deficit/hyperactivity dfisorder: Does it really tell us anything? Appl Neuropsychol Child 4(3): 188-196.

17. Banich MT, Mackiewicz KL, Depue BE, Whitmer AJ, Miller GA (2009) Cognitive control mechanisms, emotion and memory: a neural perspective with implications for psychopathology. Neurosci Biobehav Rev 33(5): 613-630.

18. Wiguna T, Guerrero AP, Wibisono S, Sastroasmoro S (2014) The amygdala's neurochemical ratios after 12 weeks administration of 20 mg long-acting methylphenidate in children with attention deficit and hyperactivity disorder: a pilot study using (1)h magnetic resonance spectroscopy. Clin Psychopharmacol. Neurosci 12(2): 137-141.

19. Sebastian A, Jung P, Krause Utz A, Lieb K, Schmahl C (2014) Frontal dysfunctions of impulse control - a systematic review in borderline personality disorder and attention-deficit/hyperactivity disorder. Front Hum Neurosci 8: 698.

20. Sachana M, Rolaki A, Bal Price A (2018) Development of the Adverse Outcome Pathway (AOP): Chronic binding of antagonist to N-methyld-aspartate receptors (NMDARs) during brain development induces impairment of learning and memory abilities of children. Toxicol, Appl Pharmacol 354: 153-175.

21. Shimizu VT, Bueno OF, Miranda MC (2014) Sensory processing abilities of children with ADHD. Braz. J Phys Ther 18(4): 343-352.

22. Amso D, Scerif G (2015) The attentive brain: insights from developmental cognitive neuroscience. Nat Rev Neurosci 16(10): 606-619.

23. Gau SS, Tseng WL, Tseng WY, Wu YH, Lo YC (2014) Association between microstructural integrity of frontostriatal tracts and school functioning: ADHD symptoms and executive function as mediators. Psychol Med 45(3): 529-543.

24. Ota T, Iida J, Nakanishi Y, Sawada S, Matsuura H, et al. (2015) Increased prefrontal hemodynamic change after atomoxetine administration in pediatric attention deficit/hyperactivity disorder as measured by nearinfrared spectroscopy. Psychiatry Clin. Neurosci. 69(3): 161-170.

25. Castejón OJ (2018) Neural correlates of cortico-thalamic-stratiattal and limbic pathways in ADHD. (Abstract) Venezuelan J Neurosci 1: 120.

26. Rubia K, Alegria AA, Brinson H (2014) Brain abnormalities in attentiondeficit hyperactivity disorder: a review. Rev Neurol 58(1): 3-18.

27. Shaw P, Sudre G, Wharton A, Weingart D, Sharp W, et al. (2014) White matter microstructure and the variable adult outcome of childhood attention deficit hyperactivity disorder. Neuropsychopharmacology 40(3): 746-754.

28. Hong SB, Harrison BJ, Fornito A, Sohn CH, Song IC, Kim JW. (2014) Functional dysconnectivity of corticostriatal circuitry and differential response to methylphenidate in youth with attention-deficit/ hyperactivity disorder. J Psychiatry Neurosci 40(1): 46-57.

29. Wu Z, Wang N, Qian Q, Yang L, Qian Y, et al. (2014) Memory characteristic in boys with attention deficit/hyperactivity disorder comorbid learning disability. Zhonghua Yi Xue Za Zhi 94(22): 1701-1704.

30. Lycett K, Mensah FK, Hiscock H, Sciberras E (2014) A prospective study of sleep problems in children with ADHD. Sleep Med 15(11): 1354-1361.

31. Dere E, Pause BM, Pietrowsky R (2010) Emotion and episodic memory in neuropsychiatric disorders. Behav. Brain Res 215(2): 162-171.

32. Gathercole SE, Woolgar F, CALM Team, Kievit RA, Astle D, Manly T, Holmes J (2016) How common are WM deficits in children with difficulties in reading and mathematics? J Appl Res Mem Cogn 5(4): 384-394. 
ISSN: 2574-1241

DOI: 10.26717/BJSTR.2019.18.003115

Orlando J Castejón. Biomed J Sci \& Tech Res

cC (P) This work is licensed under Creative BY Commons Attribution 4.0 License

Submission Link: https://biomedres.us/submit-manuscript.php

$\begin{array}{ll}\text { BIOMEDICAL } & \text { Assets of Publishing with us } \\ \text { RESEARCHES } & \text { - Global archiving of articles } \\ & \text { - Immediate, unrestricted online access } \\ & \text { - Rigorous Peer Review Process } \\ \end{array}$

